\title{
Bilateral Hypertensive Retinopathy Complicated with Retinal Neovascularization: Panretinal Photocoagulation or Intravitreal Anti-VEGF Treatment?
}

\author{
Odysseas Georgiadis Stamatina A. Kabanarou Georgios Batsos \\ Elias Feretis Tina Xirou \\ Retina Department, Red Cross Hospital, Athens, Greece
}

\section{Key Words}

Hypertensive retinopathy - Retinal neovascularization - Panretinal photocoagulation .

Anti-VEGF

\begin{abstract}
Purpose: To present the case of a patient with bilateral hypertensive retinopathy complicated with retinal neovascularization who received anti-VEGF intravitreal injection in one eye and panretinal photocoagulation (PRP) in the fellow eye. Methods: A 33-year-old male patient presented with gradual visual loss in both eyes for the last 5 months. At that time, he was examined by an ophthalmologist and occlusive retinopathy due to malignant systematic hypertension was diagnosed. He was put on antihypertensive treatment but no ophthalmic treatment was undertaken. At presentation, 5 months later, best-corrected visual acuity (BCVA) was 0.1 in the right eye (RE) and 0.9 in the left eye (LE). Fundus examination was compatible with hypertensive retinopathy complicated with retinal neovascularization. Fluorescein angiography (FFA) revealed macular ischemia mainly in the RE and large areas of peripheral retinal ischemia and neovascularization with vascular leakage in both eyes. The patient was treated with two anti-VEGF (ranibizumab) injections with 2 months interval in the RE and PRP laser in the LE. Results: Follow-up examination after 12 months showed mild improvement in BCVA, and FFA documented regression of retinal neovascularization in both eyes. Conclusion: Hypertensive retinopathy can be rarely complicated with retinal neovascularization. Treatment with PRP can be undertaken. In our case, the use of an intravitreal antiVEGF agent seemed to halt its progression satisfactorily.


Georgiadis et al.: Bilateral Hypertensive Retinopathy Complicated with Retinal Neovascularization: Panretinal Photocoagulation or Intravitreal Anti-VEGF Treatment?

\section{Introduction}

According to the World Health Organization (WHO), hypertension is the greatest risk factor for premature death worldwide, with the number of individuals with hypertension rising to 1.56 billion by 2025 [1]. Elevated blood pressure may cause vascular damage to a number of target tissues, leading to coronary heart disease, cerebral vascular disease, renal disease and retinopathy.

Hypertensive retinopathy is characterized by a spectrum of changes of the retinal vessels as a response to systematic hypertension, including narrowing, leakage and arteriosclerosis. The primary reaction is arteriolar narrowing, which may be focal or generalized. In sustained hypertension, the inner blood-retinal barrier is disrupted, with increased vascular permeability and leakage. The result is retinal hemorrhages, hard exudates and retinal edema, while papilledema is the hallmark of accelerated hypertension. Arteriosclerosis involves thickening of the vessel wall, histologically characterized by medial hypertrophy and hyalinization.

\section{Case Report}

A 33-year-old male presented with gradual visual loss in both eyes. From his past medical history, he reported mild psoriasis vulgaris, periodically treated with local medication, while his family medical history was irrelevant. The patient had visited an ophthalmologist 5 months before, and his Snellen best-corrected visual acuity (BCVA) was 0.1 in his right eye (RE) and 0.8 in his left eye (LE). Ophthalmological examination with fundoscopy, at that time, showed retinal exudates, retinal hemorrhages and cotton-wool spots, with no evidence of macular edema or optic disc edema, in both eyes (fig. 1a). Fluorescein angiography (FFA) showed macular ischemia in the RE, large areas of retinal ischemia and extensive areas of neovascularization in both eyes, mostly in the RE (fig. 1b, c). Fundus optical coherence tomography (OCT) revealed central macular atrophy in the RE and mild retinal nerve fiber edema in the LE. The diagnosis was severe retinopathy with extended occlusive vasculopathy of unknown cause. Further clinical and laboratory investigations led to the diagnosis of malignant hypertension caused by primary hyperreninemia leading to secondary aldosteronism and hypertension. The patient was treated with aliskirene for the hyperreninemia and nebivolol for his hypertension and his blood pressure returned to normal. At that time, no specific ophthalmological treatment was proposed.

Five months later, the patient presented to our department with persistent low vision mostly in his RE. Ophthalmological examination showed BCVA of 0.1 in his RE and 0.9 in his LE. Anterior segment examination was unremarkable and intraocular pressure was $13 \mathrm{~mm}$ $\mathrm{Hg}$ in both eyes. Fundus examination, as compared to the previous, showed regression of the exudates, cotton-wool spots and hemorrhages but persistent areas of neovascularization in both eyes, mostly in the RE. OCT examination showed central retinal thickness reduction in the $\mathrm{RE}$ and retinal thickness reduction temporally to the fovea in the LE. There was no evidence of macular edema in either eye (fig. 2a, b). FFA revealed macular ischemia in the RE and large areas of retinal ischemia and retinal neovascularization with vascular leakage, mainly across the temporal arcades, in both eyes (fig. 2c, d). Indocyanine green angiography did not reveal additional findings. Automated Humphrey perimetry showed a severe central scotoma expanding temporally in the RE and a small central scotoma with mild inferior temporal extension in the LE (fig. 2e, f). 
The patient underwent investigations with biochemical and immunological blood tests and detailed rheumatological examination. The blood sugar level was mildly raised (131 $\mathrm{mg} / \mathrm{dl}$ with HbA1c of 5.6\%), while all further blood tests were unremarkable [ANA, AntidsDNA, Anti-ENA, Anti-RNP, Anti-Sm, SSA(Ro), SSB(La), AMA, Anti-cardiolipin IgG and IgM, Anti-B2GP1 IgG and IgM, C-ANCA, P-ANCA, Anti-PR3, Anti-MPO, HLA-B5, and HLA-B27 were all negative]. The Chest Rx, ACE and Mantoux test were negative. Tests for virus infection (HAV, HBV, HCV and HIV, and VDRL) were negative. The patient's blood pressure was controlled $(120 / 80 \mathrm{~mm} \mathrm{Hg})$ with nebivolol and aliskirene.

Since primary fundoscopy was compatible with hypertensive retinopathy complicated with occlusive vasculopathy, and complete investigation for inflammatory disease was negative, we decided to treat the neovascularization in both eyes according to the symptoms. Anti-VEGF (ranibizumab) was injected intravitreally in the RE (to preserve its remaining peripheral vision), and panretinal photocoagulation (PRP Argon Laser) was applied in the LE (to preserve its efficient central vision).

At follow-up examination 1 month postoperatively, the patient had mild improvement of vision in both eyes. BCVA of the RE was 0.2 and BCVA of the LE was 0.9. FFA showed regression of retinal neovascularization mainly in the $\mathrm{RE}$, while in the LE there were still small areas of vascular leakage across the temporal arcades (fig. 3a, b). OCT did not show any change in either eye.

During the second follow-up visit 1 month later, the patient's BCVA was found stable, but the FFA revealed recurrence of neovascularization in the RE, mostly at the superior temporal arcade, while the LE appeared relatively stable, with small areas of vascular leakage during the late phases (fig. 3c, d). OCT examination showed no change regarding the previous status of retinal thickness. At this stage, a further anti-VEGF intravitreal injection (ranibizumab) was given to the RE and Argon laser was applied to the LE, focused on the leaking areas.

Subsequent follow-up examinations on monthly intervals for the next 10 months showed unchanged visual acuity in both eyes. FFA at 12 months showed regression of the neovascularization in the RE and regression of the vascular leakage in the LE (fig 3e, f).

\section{Discussion}

The laboratory work-up of a patient with retinal neovascularization should be based on a differential diagnosis derived from a detailed history and clinical examination, to find the underlying systemic disease.

Our patient reported suffering from mild psoriasis vulgaris, which was well controlled with occasional use of external medication. There have been some reports that relate psoriasis with intraocular inflammation [2-4]. These studies conclude that only severe pustular psoriasis and psoriasis arthropathy can be associated with uveitis and mostly with anterior segment inflammation. Posterior uveitis with retinal disease that varies from edema and exudates to occlusive vasculopathy appears less often.

Systematic diseases such as autoimmune rheumatic diseases (systemic lupus erythematosus, antiphospholipid antibody syndrome, preeclampsia/eclampsia syndrome, drugrelated and other vasculopathies) could be the precipitating cause of retinal vasculopathy that can lead to retinal ischemia and secondary neovascularization [5-7]. Our patient was thoroughly investigated, and the possibility of autoimmune rheumatic disease was excluded by negative biochemical and immunological examinations. Therefore, his ischemic retinopa- 
thy was attributed to systematic malignant hypertension due to idiopathic hyperreninemia and secondary hyperaldosteronism.

Ischemia, inadequate supply of oxygen and subsequent elevation of angiogenic factors are thought to be major and important contributors in retinal neovascularization, in the natural course of diseases such as diabetic retinopathy and retinal vascular occlusions. The role of VEGFs in particular is well established and so is the effectiveness of anti-VEGF treatment in the inhibition of ischemic related ocular neovascularization. Anti-VEGF treatment for persistent retinopathy secondary to malignant hypertension has been reported $[8,9]$. Whereas there is no reference of neovascularization in these studies, intravitreal anti-VEGF (bevacizumab) in addition to proper antihypertensive medical treatment was found to be an effective and safe approach to succeed regression of vascular leakage, macular edema and optic disc edema.

Since the appearance of anti-VEGF agents in the management of ocular diseases, there is a trend to expand the spectrum of their indications. Currently, VEGF inhibitors appear to be the most promising treatment modality for choroidal neovascularization secondary to agerelated macular degeneration, myopia, and the management of diabetic macular edema.

Ranibizumab is a humanized murine monoclonal antibody fragment (Fab), much smaller than the parent molecule and affinity purified to provide stronger binding and inhibition of a number of isoforms of VEGF-A. Blocking VEGF-A can restrain abnormal new blood vessel formation and decrease the resultant vascular leakage. At present, a respectful number of randomized clinical trials have established official indication of ranibizumab for wet age-related macular degeneration, diabetic macular edema, retinal vein occlusion, and lately myopic choroidal neovascularization [10].

The existing case series reporting on the use of VEGF inhibitors have consistently demonstrated significant improvement in vision along with negligible short-term safety concerns. A subject for consideration should be the effect of VEGF inhibitors on vascular integrity, especially after repeated intraocular anti-VEGF injections [11].

To date, several studies have reported panretinal laser photocoagulation as the most indicated treatment for retinal neovascularization secondary to proliferative diabetic retinopathy [12], or secondary to branch retinal vein occlusion and central retinal vein occlusion $[13,14]$. Although PRP is currently the only approved treatment for the management of peripheral retinal ischemia complicated with retinal neovascularization, ocular complications such as impaired night vision, diminished visual field and reduced color vision and contrast sensitivity should not be overlooked [15].

In the presented case, we treated the neovascularization in the LE, with two sessions of PRP, 2 months apart, and we used an anti-VEGF agent for the RE, which had the most severe retinal damage and central visual loss but maintained adequate peripheral vision. Two intravitreal injections were administered, the first one upon presentation and the second 2 months later. Stabilization of visual acuity and regression of neovascularization was noted in both eyes for a follow-up period of 12 months.

\section{Conclusion}

Hypertensive retinopathy can be rarely complicated with retinal neovascularization. Treatment with PRP has been the treatment of choice for many years. However, reported problems with peripheral vision restriction initiate the necessity for exploring alternative treatment options. Our approach of using ranibizumab could trigger further research 
towards the evaluation of anti-VEGF agents in the management of retinal ischemia and neovascularization.

\section{Disclosure Statement}

The authors have no proprietary interest. There was no financial support for this study.

\section{References}

1 Kearney PM, Whelton M, Reynolds K, Muntner P, Whelton PK, He J: Global burden of hypertension analysis of worldwide data. Lancet 2005;365:217-223.

-2 Fraga NA, Oliveira Mde F, Follador I, Rocha Bde O, Rêgo VR: Psoriasis and uveitis: a literature review. An Bras Dermatol 2012;87:877-883.

3 Knox DL: Psoriasis and intraocular inflammation. Trans Am Ophthalmol Soc 1979;77:210-224.

-4 Rehal B, Modjtahedi BS, Morse LS, Schwab IR, Maibach HI: Ocular psoriasis. J Am Acad Dermatol 2011;65:1202-1212.

5 Giorgi D, Pace F, Giorgi A, Bonomo L, Gabrieli CB: Retinopathy in systematic lupus erythymatosus: pathogenesis and approach to therapy. Hum Immunol 1999;60:688-696.

-6 Jabs DA, Fine SL, Hochberg MC, Newman SA, Heiner GG, Stevens MB: Severe retinal vaso-occlusive disease in systemic lupus erythematosus. Arch Ophthalmol 1986;104:558-563.

-7 Asherson RA, Merry P, Acheson JF, Harris EN, Hughes GR: Antiphospholipid antibodies: a risk factor for occlusive ocular vascular disease in systematic lupus erythematosus and the 'primary' antiphospholipid syndrome. Ann Rheum Dis 1989;48:358-361.

8 Abdelrahman Gaber Salman: Intravitreal bevacizumab in persistent retinopathy secondary to malignant hypertension. Saudi J Ophthalmol 2013;27:25-29.

-9 Kim EY, Lew HM, Song JH: Effect of intravitreal bevacizumab (Avastin) therapy in malignant hypertensive retinopathy: a report of two cases. J Ocul Pharmacol Ther 2012;28:318-322.

10 Wolf S, Balciuniene VJ, Laganovska G, Menchini U, Ohno-Matsui K, Sharma T, Silva R, Pilz S, Gekkieva M, RADIANCE Study Group; RADIANCE: a randomized controlled study of ranibizumab in patients with choroidal neovascularization secondary to pathologic myopia. Ophthalmology 2014;121:682-692.

-11 Manousaridis K, Talks J: Macular ischaemia: a contraindication for anti-VEGF treatment in retinal vascular disease? Br J Ophthalmol 2012;96:179-184.

12 Early Treatment Diabetic Retinopathy Study Research Group: Early photocoagulation for diabetic retinopathy. ETDRS report number 9. Ophthalmology 1991;98(suppl 5):766-785.

13 The Central Retinal Vein Occlusion Study Group N report: A randomized clinical trial of early panretinal photocoagulation for ischemic central retinal vein occlusion. Ophthalmology 1995;102:1434-1444.

14 Branch Vein Occlusion Study Group: Argon laser scatter photocoagulation for prevention of neovascularization and vitreous hemorrhage in branch vein occlusion. A randomized clinical trial. Arch Ophthalmol 2008;126:513-518.

15 Henricsson M, Heijl A: The effect of panretinal laser photocoagulation on visual acuity, visual fields and on subjective visual impairment in preproliferative and early proliferative diabetic retinopathy. Acta Ophthalmol (Copenh) 1994;72:570-575. 
Georgiadis et al.: Bilateral Hypertensive Retinopathy Complicated with Retinal

Neovascularization: Panretinal Photocoagulation or Intravitreal Anti-VEGF Treatment?
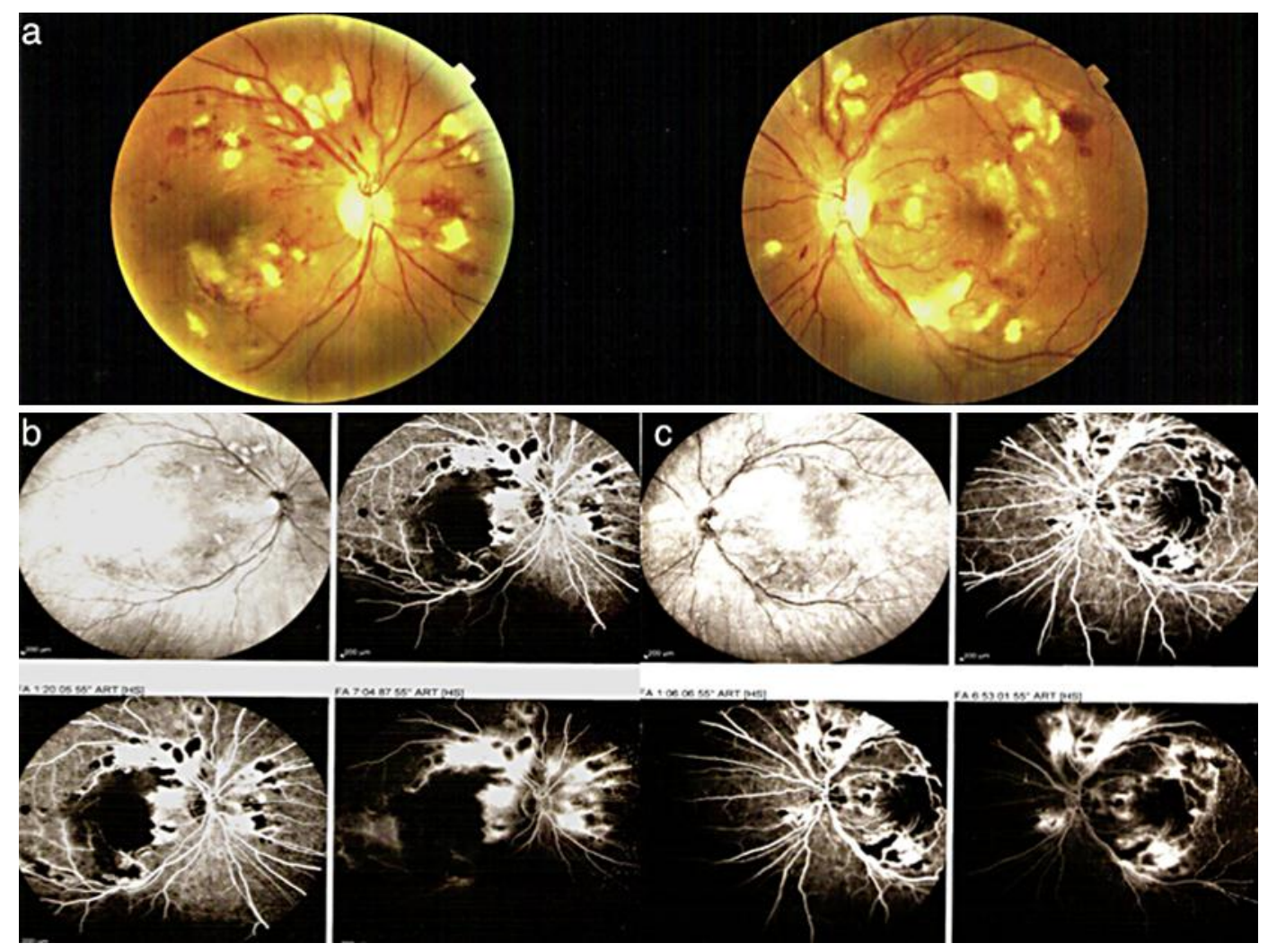

Fig. 1. a Color fundus photographs at initial examination showing retinal exudates, retinal hemorrhages and cotton-wool spots in both eyes. b, c FFA showing macular ischemia in the RE, large areas of retinal ischemia in both eyes and extensive areas of neovascularization in both eyes, mostly in the RE. 


\section{Case Reports in \\ Ophthalmology}

\begin{tabular}{l|l}
\hline Case Rep Ophthalmol 2014;5:231-238 \\
\hline DOI: 10.1159/000365865 & $\begin{array}{l}\text { ○ 2014 S. Karger AG, Basel } \\
\text { www.karger.com/cop }\end{array}$ \\
\hline
\end{tabular}

(C) 2014 S. Karger AG, Base

Georgiadis et al.: Bilateral Hypertensive Retinopathy Complicated with Retinal

Neovascularization: Panretinal Photocoagulation or Intravitreal Anti-VEGF Treatment?

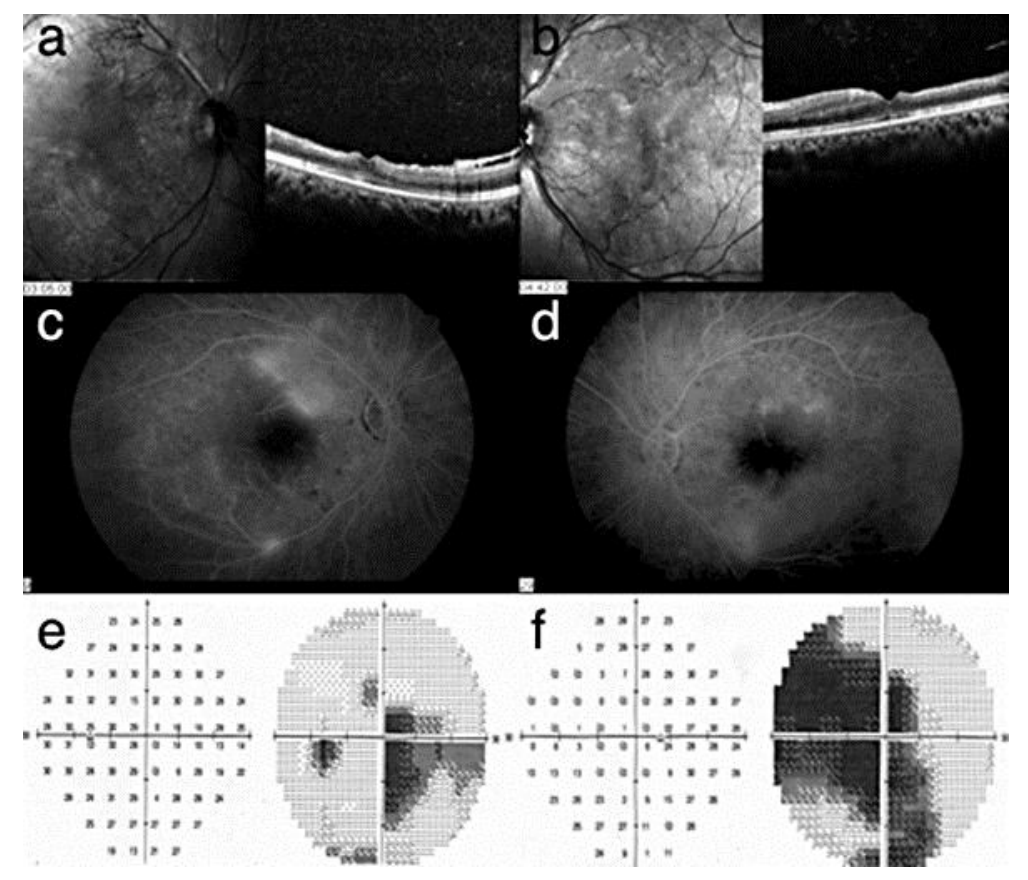

Fig. 2. a, b OCT at presentation to our clinic showing reduction of central retinal thickness in the RE and retinal thickness reduction temporally to the fovea in the LE. c, $\mathbf{d}$ FFA showing macular ischemia in the RE and large areas of retinal ischemia and retinal neovascularization with vascular leakage, mainly across the temporal arcades, in both eyes. e, $\mathbf{f}$ Automated Humphrey perimetry showing a severe central scotoma expanding temporally in the RE and a small central scotoma with mild inferior temporal extension in the LE. 
Case Reports in

Ophthalmology

\section{Case Rep Ophthalmol 2014;5:231-238}

DOI: $10.1159 / 000365865$

Georgiadis et al.: Bilateral Hypertensive Retinopathy Complicated with Retinal

Neovascularization: Panretinal Photocoagulation or Intravitreal Anti-VEGF Treatment?

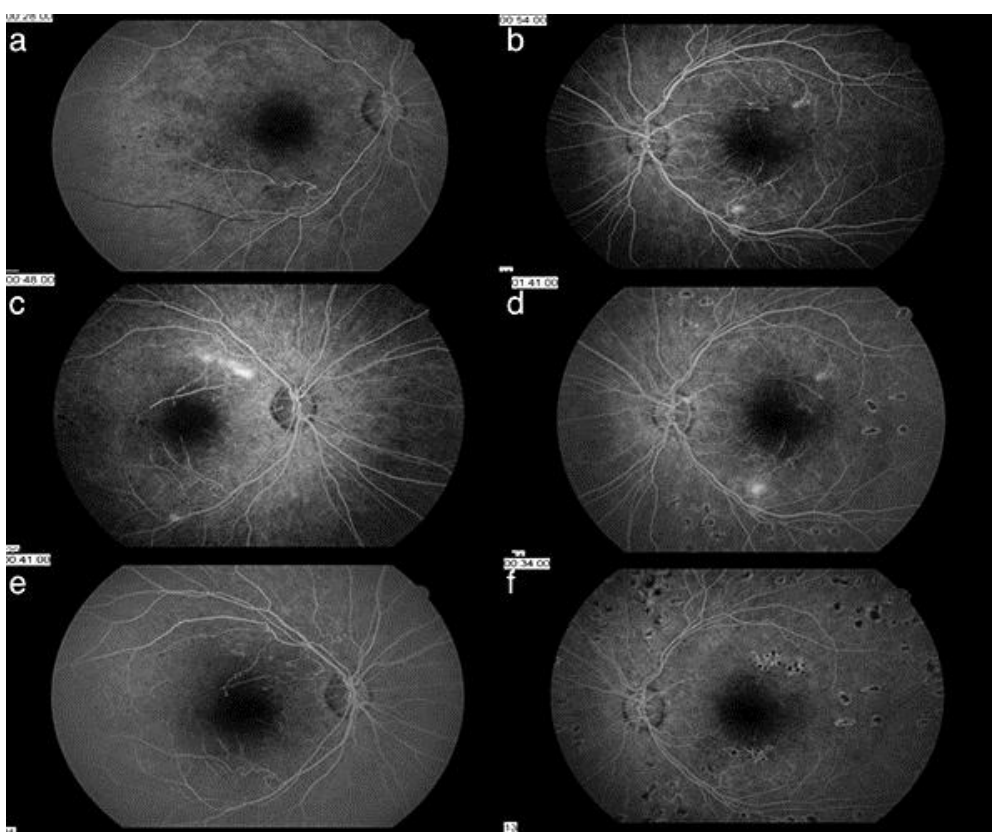

Fig. 3. a, b FFA at follow-up 1 month postoperatively showing regression of retinal neovascularization mainly in the RE and small areas of vascular leakage remaining in the LE. c, $\mathbf{d}$ FFA at the second follow-up visit showing recurrence of neovascularization at the superior temporal arcade in the RE, while the LE appears relatively stable, with small areas of vascular leakage during the late phases. e, f FFA at 12 months showing regression of the neovascularization in the RE and regression of the vascular leakage in the LE. 